\title{
Hypercoagulability in Liver Transplant Recipients: Does Portal Vein Thrombosis Predict Post-Operative Thrombotic Complications?
}

\author{
Brintha K. Enestvedt ${ }^{1}$, C. Kristian Enestvedt ${ }^{2}$, Brian Diggs ${ }^{3}$, Susan L. Orloff ${ }^{4}$ \\ ${ }^{1}$ Division of Gastroenterology, The University of Pennsylvania, Philadelphia, USA \\ ${ }^{2}$ Division of Abdominal Transplantation, The University of Pennsylvania, Philadelphia, USA \\ ${ }^{3}$ Department of Surgery, Oregon Health \& Science University, Portland, USA \\ ${ }^{4}$ Abdominal Transplantation, Oregon Health \& Science University \\ and Portland Veteran's Affairs Medical Center, Portland, USA \\ E-mail: brintha.enestvedt@uphs.upenn.edu \\ Received July 30, 2011; revised August 15, 2011; accepted August 23, 2011
}

\begin{abstract}
Background: Cirrhotic patients have higher rates of hypercoagulable disorders. We hypothesized that orthotopic liver transplant (OLT) recipients with pre-operative portal vein thrombosis (PVT) have more postoperative thrombotic events than those without PVT. Aims: To compare rates of post-op thrombotic events and outcomes between those with and without pre-op PVT. Methods: All OLT recipients between 1/02-4/09 were retrospectively reviewed. Outcome measures included survival, deep venous thrombosis, pulmonary embolism, hepatic artery thrombosis, and recurrent PVT. Minimum follow up was 6 months. Results: In 363 OLTs performed, mean recipient age was $53.1 \mathrm{yrs}( \pm 9.2) ; 268$ patients were male. Mean MELD at transplant was 22.1 ( \pm 6.2$)$. The prevalence of pre-op PVT was $11.2 \%(41 / 350)$. There was no difference in the $\%$ of post-op thrombotic events between those with and without PVT $(\mathrm{p}=0.77)$. MELD, recipient and donor age, and gender were similar in both groups. Mean survival in those with pre-op PVT was 85.2 months vs. 78.7 in those without PVT $(p=0.19)$. Conclusions: The rate of post-op thrombotic events was equivalent in OLT recipients with and without pre-op PVT. The presence of PVT did not adversely impact patient survival and should not be a contraindication to OLT.
\end{abstract}

Keywords: Portal Vein Thrombosis, Orthotopic Liver Transplantation, Hypercoagulability, Thromboses

\section{Introduction}

Portal vein thrombosis is a known complication in those with advanced end stage liver disease (ESLD), occurring in $0.6 \%-25 \%$ [1-4] of these patients in contemporary reports. In the largest study to date a prevalence of $11 \%$ was reported in 701 patients with cirrhosis [5]. Once considered an absolute contraindication to orthotopic liver transplantation (OLT), portal vein thrombosis (PVT) can be managed operatively with thrombectomy or venous replacement conduits; however due to the surgical complexity and high risk for recurrence, it remains a formidable clinical problem [2,6-10]. While mostly associated with hepatocellular carcinoma, PVT in cirrhotic patients, even in the absence of neoplasia may be the result of an underlying hypercoagulable disorder, as is suggested by some preliminary data [11-13].

Mounting evidence suggests that patients with cirrhosis, similar to those with pulmonary embolism or deep venous thrombosis, have underlying inherited or acquired thrombophilic disorders such as prothrombin gene mutation, hyperhomocysteinemia (MTHFR gene mutation), anti-cardiolipin antibodies or Factor V Leiden [4-5, 14-17]. This hypercoagulable condition may also contribute to post-operative thrombotic events which can be devastating and life threatening. The identification prior to OLT of a pre-operative risk factor for post-operative clot formation (such as portal vein thrombosis), would enable the institution of preventive strategies to limit these thrombotic complications. Unfortunately, the available literature on PVT in cirrhotics is limited by cross sectional studies with small sample sizes and few controlled data on 
which to base clinical decisions in patients with PVT.

We hypothesize that liver transplant recipients with pre-operative portal vein thrombosis have a higher rate of post operative thrombotic events than those without PVT. The aims of the current study were to describe the prevalence of pre-operative portal vein thrombosis in liver transplant recipients, describe intra-operative management particular to this problem, and compare the rates of post-operative thrombotic events and overall outcomes between those with and without pre-operative PVT. We also evaluated significant risk factors for the development of portal vein thrombosis and other postoperative clot development.

\section{Materials and Methods}

A retrospective chart review was performed of all patients who underwent OLT between 1/02- 4/09. Data gathered included recipient and donor age and gender, Model for End Stage Liver Disease (MELD) score, etiology of ESLD, presence of hepatocellular carcinoma (HCC), PVT at the time of liver transplant, warm and cold ischemia times, CMV donor-recipient status, use of donor after cardiac death (DCD) organ, intra-operative management of PVT (thrombectomy, iliac conduit), the development of post operative thromboses (including deep venous thrombosis, pulmonary embolism, hepatic artery thrombosis, and recurrent PVT), post operative biliary stricture, rejection, bile leak and survival. The diagnosis of portal vein thrombosis was made either by pre-operative imaging by computer tomography (all patients underwent immediate pre-operative CT scans), ultrasound or by intra-operative findings.

The primary outcome measures were post-operative thrombotic events such as recurrent PVT, hepatic artery thrombosis, PE and DVT as confirmed by radiographic studies. Principal diagnostic tests included Duplex ultrasound of either the hepatic vasculature or the lower extremities (for DVT) or contrast CT of the chest (for PE) or of the abdomen (for PVT). These imaging modalities were pursued for symptoms. Secondary outcomes included post-operative biliary stricture, leak, rejection and survival (measured in months). All transplants were performed at a single shared transplant center consisting of Oregon Health \& Science University (OHSU) and the Portland Veteran's Affairs Medical Center (PVAMC) and the pre-, peri- and post-operative care was managed by this shared transplant institution's staff.

The UNOS listed MELD was used for analyses except for those patients who had a MELD exception for HCC. In these patients biologic MELD was substituted to better reflect their true liver disease severity. We excluded patients with Status 1 priority as their physiology is not likely related to underlying cirrhosis. All patients had a minimum of 6 months of follow up. Univariate statistical analyses included student's t-test, $\mathrm{X}^{2}$ test, and Kaplan Meier log-rank were performed using SPSS version 17.0 (SPSS Inc, Chicago, IL). Binary logistic regression was performed to identify risk factors for post-operative clot. A $\mathrm{p}$ value of $<0.2$ on univariate analysis met inclusion in multivariate analysis. A $p$ value $<0.05$ was significant on multivariate analyses. Covariates included in the multivariate model included all significant univariate variables in addition to those variables deemed clinically relevant to the development of post operative clot. This study was approved by the IRB committees at Oregon Health \& Science University and the Portland Veteran's Affairs Medical Center.

\section{Results}

A total of 976 orthotopic liver transplants were performed at our institution from 1998 to April, 2009. Three hundred and sixty two (362) occurred during the study time frame (2/02-4/09). After excluding patients with status 1 priority $(\mathrm{n}=12)$, the study cohort was 350 patients. Table 1 describes the demographics of the study cohort and compares those with and without portal vein thrombosis. The overall mean recipient age was 53.1 \pm 9.2 years old. The majority of OLT recipients were male (76.6\%); the mean MELD was $22.1 \pm 6.23$. Alcohol $(\mathrm{ETOH})$ and hepatitis $\mathrm{C}(\mathrm{HCV})$ comprised $65 \%$ of the etiologies of ESLD. Hepatocellular carcinoma (HCC) was found in 108 patients $(30.9 \%)$. The complete distribution of the etiology of end stage liver disease (ESLD) is as follows: $\mathrm{HCV}$ alone $17.4 \%(61), \mathrm{ETOH} / \mathrm{HCV}$ $16.6 \%$ (58), $\mathrm{HCV} / \mathrm{HCC} 14.3 \%$ (50), PSC 10.6\% (37), ETOH alone 6.9\% (24), ETOH/HCV/HCC 6.6\% (23), HBV related 5.1\% (18), PBC 4.9\% (17), NASH 3.4\% (12), ETOH/HCC 3.1\% (11), Autoimmune hepatitis $2.9 \%$ (10), Cryptogenic cirrhosis $2.6 \%$ (9), other $5.7 \%$ (20).

The prevalence of portal vein thrombosis was $11.2 \%$ $(\mathrm{n}=41) ; 77.5 \%(\mathrm{n}=31)$ were partial thromboses and 9 patients had complete PVTs. There was no difference in the recipient or donor age and gender, or MELD between those with and without pre-operative PVT (Table 1). The post-operative thrombotic rate in those patients with pre-operative portal vein thrombosis was $9.8 \%(4 / 41)$ compared with $8.4 \%(26 / 309)$ in those without PVT ( $p=$ 0.77, Table 2). There was no difference in the type of post-operative thrombosis between the two groups. Of all the etiologies of ESLD, only alcohol related liver disease was statistically significantly higher in those who had PVT compared to those without $(48.8 \%$ vs. $32.7 \%, \mathrm{p}=$ 
Table 1. Demographics of patients with and without pre-operative portal vein thrombosis.

\begin{tabular}{|c|c|c|c|c|}
\hline & All OLTs & PVT & No PVT & $p$ value \\
\hline N (\%) & $350(100 \%)$ & $41(11.2 \%)$ & $309(88.3 \%)$ & \\
\hline Mean Recipient Age (yrs) & $53.1 \pm 9.2$ & $55.2 \pm 8.6$ & $52.8 \pm 9.5$ & 0.12 \\
\hline Recipient Gender & $\begin{array}{c}\text { M } 268(76.6 \%) \\
\text { F } 82(23.4 \%)\end{array}$ & $\begin{array}{c}\text { M } 31(75.6 \%) \\
\text { F } 10(24.4 \%)\end{array}$ & $\begin{array}{c}\text { M } 237(76.7 \%) \\
\text { F } 71(23.3 \%)\end{array}$ & 0.88 \\
\hline MELD & $22.11 \pm 6.2$ & $21.0 \pm 5.4$ & $22.3 \pm 6.8$ & 0.25 \\
\hline Mean Donor Age (yrs) & $38.9 \pm 16.7$ & 42.6 & 38.4 & 0.20 \\
\hline Donor Gender & $\begin{array}{c}\text { M } 207(60.7 \%) \\
\text { F } 134(39.3 \%)\end{array}$ & $\begin{array}{c}\text { M } 24(58.5 \%) \\
\text { F } 7(39.0 \%)\end{array}$ & $\begin{array}{c}\text { M } 183(59.4 \%) \\
\text { F } 117(38.0 \%)\end{array}$ & 0.87 \\
\hline
\end{tabular}

OLT: orthotopic liver transplant; PVT: portal vein thrombosis; MELD: model for end stage liver disease.

Table 2. Prevalence of post operative thromboses.

\begin{tabular}{cccccc}
\hline & \multicolumn{5}{c}{ Post Operative Thromboses } \\
\hline & PVT & Other Clot & Hepatic Artery Thrombosis & Total Clots & p value \\
Pre-Op PVT (41) & 2 & 2 & 0 & $4(9.8 \%)$ & 0.77 \\
No Pre-Op PVT (309) & 4 & 16 & 6 & $26(8.4 \%)$ & 30 \\
Total (350) & 6 & 18 & 6 & 0.37 & \\
p value & 0.10 & 0.93 & & \\
\hline
\end{tabular}

PVT $=$ portal vein thrombosis.

0.042). Operative thrombectomy was performed in $68 \%$ (28) of patients with PVT.

The rates of rejection, biliary stricture, biliary leak and need for re-transplantation were not significantly different between those with and without PVT (Table 3). Median survival in those with PVT was 82.5 months compared with 78.7 months in those without PVT $(\mathrm{p}=0.19$. Figure 1).

Thirty patients $(8.6 \%, 30 / 350)$ developed a postoperative clot. The following variables were included in a multivariate regression model assessing risk factors for post operative clot: recipient age, recipient gender, etiology of ESLD, MELD, pre-op PVT, donor age, donor gender, warm and cold ischemia time, CMV risk status (low risk, intermediate risk, high risk), DCD liver, HCC, rejection (Tables 4(a) and (b)). Female donor gender was associated with a three-fold greater risk of postoperative clot compared to male donors (OR 3.06, 95\% CI $1.14-8.24, p=0.027$ ) (Table 4(b)). Increasing warm ischemia time was associated with post-operative clot (OR 1.05, 95\% CI 1.01-1.09, $\mathrm{p}=0.016$ ) for each minute of warm ischemia time, the post-operative clot risk increased by $5 \%$. Rejection was associated with a threefold increase in post-operative clot (OR 3.36, 95\% CI $1.21-9.37, \mathrm{p}=0.020)$. DCD livers were associated with the highest risk for post-operative thrombotic complications (OR 7.49, 95\% CI 1.03 - 54.28, p = 0.046). Inter- mediate CMV status (donor negative, recipient positive) was inversely associated with post-operative thrombosis (OR 0.07, 95\% CI $0.07-0.81, \mathrm{p}=0.034$ ).

\section{Discussion}

In this study at a single shared transplant center, the prevalence of pre-operative portal vein thrombosis in liver transplant recipients was $11.2 \%$ which is similar to

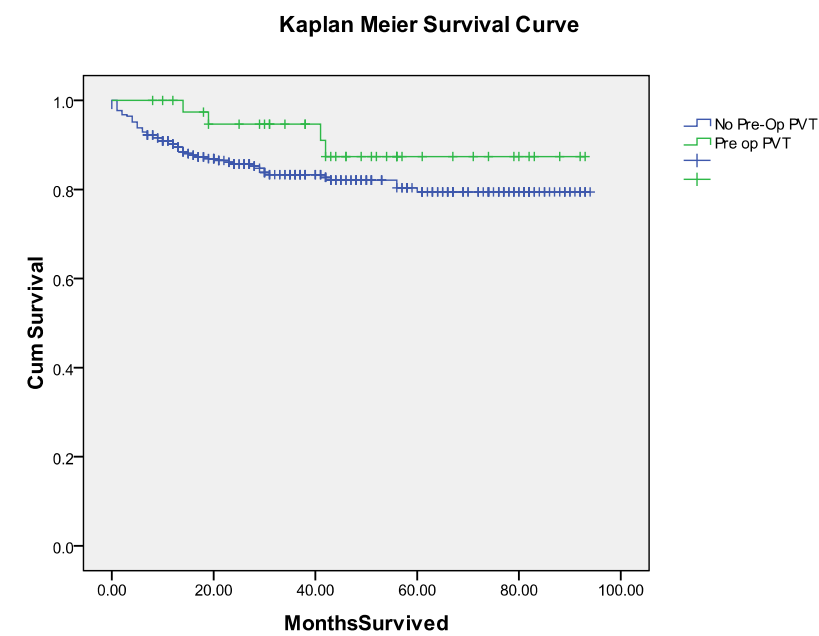

Figure 1. Median survival post orthotopic liver transplantation. 
Table 3. Prevalence of non thrombotic post operative outcomes.

\begin{tabular}{cccc}
\hline Post Operative Outcomes & PVT (41) & No PVT (309) & p value \\
\hline Rejection & $9(22 \%)$ & $63(20.6 \%)$ & 0.84 \\
Biliary Stricture & $11(26.8 \%)$ & $51(16.7 \%)$ & 0.11 \\
Biliary Leak & $2(5.0 \%)$ & $13(4.2 \%)$ & 0.83 \\
Re-Transplant & $1(2.4 \%)$ & $12(3.9 \%)$ & 0.65 \\
\hline
\end{tabular}

Table 4. (a) Risk factors for post operative thrombosis, univariate analysis (significant $\mathbf{p}<0.20$ ); (b) Significant risk factors for post operative thrombosis, multivariate analysis.

(a)

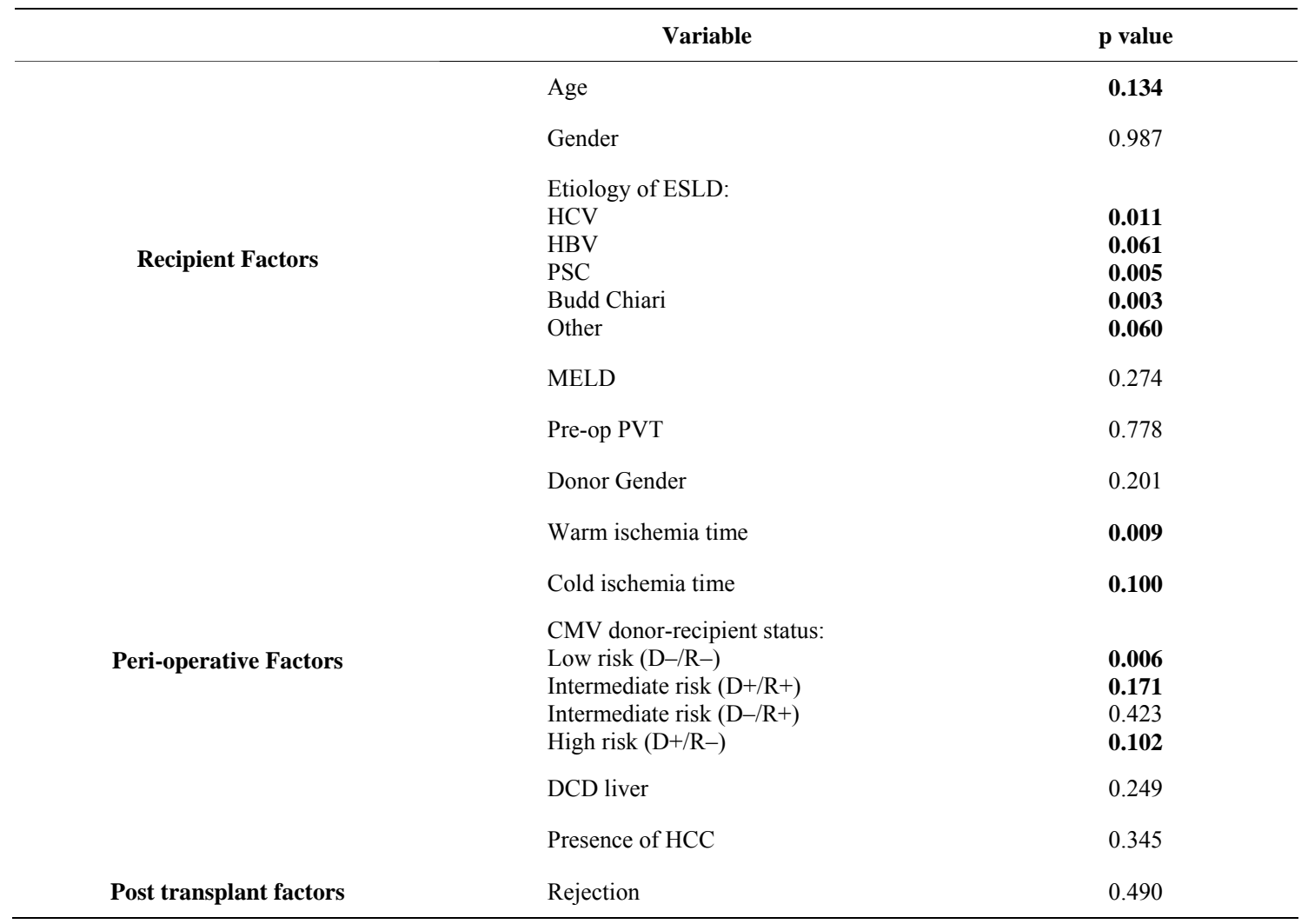

$\mathrm{N}=30$; DCD: donor after cardiac death; $\mathrm{D}-/ \mathrm{R}-$ : donor negative $\mathrm{CMV}$, recipient negative CMV.

(b)

\begin{tabular}{lcccc}
\hline & Variable & p value & Odds ratio & 95\% CI \\
\cline { 2 - 5 } & Donor Gender & 0.027 & 3.06 & $1.14-8.24$ \\
Peri-operative factors & Warm ischemia time & 0.016 & $1.05^{\neq}$ & $1.01-1.09$ \\
& Cold ischemia time & 0.015 & $1.00^{\neq}$ & $0.99-1.00$ \\
Pos donor-recipient status Intermediate risk (D-/R+) & 0.034 & 0.07 & $0.07-0.81$ \\
\hline \multirow{2}{*}{ Post transplant factors } & DCD liver & 0.046 & 7.49 & $1.03-54.28$ \\
\hline
\end{tabular}

$\neq$ per minute of ischemia time. 
that previously reported $[1,3-6,18]$. While PVT was once considered a contraindication to liver transplantation and thus significantly impacted a patient's survival, advances in surgical technique (thrombectomy, venoplasty and iliac vein conduit) and catheter-directed therapies have enabled successful liver transplantation in these patients. However, PVT continues to pose clinical challenges. Emerging evidence supports the role of acquired or inherited hypercoagulability, in particular JAK-2 mutation, in the formation of portal vein thrombosis, even in patients with cirrhosis.

This study demonstrated that patients with pre-operative PVT did not have a higher rate of post operative thromboses. Therefore, patients with PVT do not need increased thrombosis surveillance in the post-operative period. An important finding in this study was that there was no significant difference in survival between those who did and did not have portal vein thrombosis. Other authors have reported decreased graft and patient survival in patients with PVT. In one of the largest reports detailing vascular complications in liver transplants, Duffy et al showed a profound decrease in graft survival for those patients who developed a postoperative PVT $(\mathrm{p}<0.001)$ [19]. The authors identified the presence of pre-operative PVT as well as the use of a portal vein graft as a significant risk factor for postoperative PVT $(p<0.01)$, In addition, these authors noted a significant 'late' thrombosis rate, which occurred in $35 \%$ of the PVT patients. However, Duffy and colleagues reported a post-transplant PVT rate of only $3 \%$ in the 215 patients for whom a pre-operative diagnosis was made and eversion thromboendovenectomy was performed, indicating that early identification and appropriate operative intervention are essential to ensure good outcomes [19]. It may be that our study has not captured a percentage of these patients who develop 'late' thromboses, due to insufficient follow up time or subclinical presentation. Additionally, the disparities in our findings may be further explained by the smaller cohort and number of post-operative PVTs, as well as improvements in operative and radiologic imaging techniques, and post-operative therapies. In particular, adequate pre-operative recognition of this problem leads to better operative planning and management of the difficult portal vein. At the time of listing patients for transplantation at our center, routine pre-operative review of all imaging is performed to prepare for intra-operative and anatomic challenges. Our findings are supported by other series that have demonstrated excellent outcomes with operative thrombectomy in patients with preop PVT $[20,21]$.

Multivariate regression analysis identified several significant risk factors for the development of post- operative clot. These included longer warm ischemia time, DCD liver, donor gender and rejection. The finding that both increased warm ischemia time and the use of DCD livers contributed to a higher complication rate is consistent with the existing body of literature. Previous authors have also shown an association between female donor gender and post-transplant complications, as supported in the present study [22-27]. It may be that the higher estrogen milieu of the female donor influences thrombotic events in the recipient; however, further study is needed to confirm this hypothesis. Further analysis of the treatment regimens used for rejection might indicate an association between thromboses and the therapeutic agents commonly used to treat rejection and could be an excellent area for further investigation.

The current study has several limitations. First, it is prone to those shortcomings inherent in a retrospective database review. Additionally, if patient follow-up occurred outside our transplant institution, some of the posttransplant thrombotic events may have been missed in the data-capture process. We have extensive follow-up and event reporting practices in place as dictated by the UNOS guidelines and our own center policies. Therefore, we estimate that the proportion of patients receiving care outside our transplant center is very low, and missed events even less common.

The thrombosis rate in our cohort reflects symptomatic or clinically relevant clots (revealed in the work up of symptoms or abnormal lab tests etc.) and therefore may be an underestimation of the true clot burden. Clinically insignificant clots, such as those that may be incidentally discovered on routine imaging were not included, as these had no clinical impact. The influence of DVT prophylaxis with heparin products in the peri-operative period on the subsequent development of post-operative thrombosis is not well understood. During the study time frame there was not a consistent DVT prophylaxis protocol in place. Additionally, we did not have a consistent policy of surveying these patients for known hypercoagulable conditions such as Factor $\mathrm{V}$ Leiden Mutations, protein $\mathrm{C}$ and $\mathrm{S}$ deficiencies, hyper-homocysteinemia, etc in pre or post transplant patient nor of consistently working up patients with PVT for these disorders. Given the growing interest in hypercoagulability in patients with liver failure and cirrhosis, a hypercoagulable serologic work-up is appropriate in the pre-operative setting, in order to gain a better understanding of the true presence of a hypercoagulable state in this patient population. The addition of these laboratory tests and the prospective study of any respective correlation in these patients will strengthen future studies.

Notwithstanding the above limitations, there is limited 
published data on this topic and the current study is strengthened by the relatively large cohort. Additionally, given the contemporary study time frame, the data reflects modern day liver transplant and portal vein thrombosis management practices, which did not exist in previous reports in the literature. Moreover, all the patients in the cohort were managed at a single shared transplant center and are more likely to have had uniformity in pre-operative, intra-operative, and post-operative clinical management. These data suggest that posttransplant thromboses are not contributing to a substantial clinical burden in the care of OLT recipients. Nonetheless, it is critical for surgeons and hepatologists to recognize the association between warm ischemia time, DCD liver, donor gender and rejection and post-transplant thromboses to ensure timely recognition and management.

In summary, pre-transplant portal vein thrombosis was not associated with post operative thromboses. In addition, pre-operative portal vein thrombosis should not preclude liver transplantation in the otherwise appropriate candidate. Given the current lack of consensus opinion or evidence based practices regarding the diagnosis and clinical management of portal vein thrombosis, future prospective studies are warranted to address this clinical entity.

\section{Acknowledgements}

Authors' contributions: Brintha K. Enestvedt: participated in research design, performance of research, data analysis and manuscript preparation; C. Kristian Enestvedt: participated in research design, performance of research, data analysis and manuscript preparation Brian Diggs: participated in statistical analysis and data interpretation; Susan L. Orloff: participated in research design, data analysis and manuscript preparation;

\section{References}

[1] M. Ogren, D. Bergqvist, M. Bjorck, S. Acosta, H. Eriksson and N. H. Sternby, "Portal Vein Thrombosis: Prevalence, Patient Characteristics and Lifetime Risk: A Population Study Based on 23,796 Consecutive Autopsies," World Journal of Gastroenterology, Vol. 12, No. 13, 2006, pp. 2115-2119.

[2] B. R. Davidson, M. Gibson, R. Dick, A. Burroughs and K. Rolles, "Incidence, Risk Factors, Management, and Outcome of Portal Vein Abnormalities at Orthotopic Liver Transplantation," Transplantation, Vol. 57, No. 8, 1994, pp. 1174-1177. doi:10.1097/00007890-199404270-00006

[3] E. A. Tsochatzis, M. Senzolo, G. Germani, A. Gatt and A. K. Burroughs, "Systematic Review: Portal Vein Thrombosis in Cirrhosis," Alimentary Pharmacology and Therapeutics, Vol. 31, No. 3, 2010, pp. 366-374. doi:10.1111/j.1365-2036.2009.04182.x

[4] L. Amitrano, V. Brancaccio, M. A. Guardascione, et al., "Inherited Coagulation Disorders in Cirrhotic Patients with Portal Vein Thrombosis," Hepatology, Vol. 31, No. 2, 2000, pp. 345-348. doi:10.1002/hep.510310213

[5] L. Amitrano, M. A. Guardascione, V. Brancaccio, et al., "Risk Factors and Clinical Presentation of Portal Vein Thrombosis in Patients with Liver Cirrhosis," Journal of Hepatology, Vol. 40, No. 5, 2004, pp. 736-741. doi:10.1016/j.jhep.2004.01.001

[6] G. Manzanet, F. Sanjuan, P. Orbis, et al., "Liver Transplantation in Patients with Portal Vein Thrombosis," Liver Transplantation, Vol. 7, No. 2, 2001, pp. 125-131. doi:10.1053/jlts.2001.21295

[7] T. Nonami, I. Yokoyama, S. Iwatsuki and T. E. Starzl, "The Incidence of Portal Vein Thrombosis at Liver Transplantation," Hepatology, Vol. 16, No. 5, 1992, pp. 1195-1198. doi:10.1002/hep.1840160515

[8] A. Shaked and R. W. Busuttil, "Liver Transplantation in Patients with Portal Vein Thrombosis and Central Portacaval Shunts," Annals of Surgery, Vol. 214, No. 6, 1991, pp. 696-702. doi:10.1097/00000658-199112000-00009

[9] A. C. Stieber, G. Zetti, S. Todo, et al., "The Spectrum of Portal Vein Thrombosis in Liver Transplantation," Annals of Surgery, Vol. 213, No. 3, 1991, pp. 199-206. doi:10.1097/00000658-199103000-00003

[10] M. J. Englesbe, J. Kubus, W. Muhammad, et al., "Portal Vein Thrombosis and Survival in Patients with Cirrhosis," Liver Transplantation, Vol. 16, No. 1, 2010, pp. 83-90. doi:10.1002/1t.21941

[11] M. H. Denninger, Y. Chait, N. Casadevall, et al., "Cause of Portal or Hepatic Venous Thrombosis in Adults: The Role of Multiple Concurrent Factors," Hepatology, Vol. 31, No. 3, 2000, pp. 587-591. doi: $10.1002 /$ hep. 510310307

[12] H. L. Janssen, J. R. Meinardi, F. P. Vleggaar, et al., "Factor V Leiden Mutation, Prothrombin Gene Mutation, and Deficiencies in Coagulation Inhibitors Associated with Budd-Chiari Syndrome and Portal Vein Thrombosis: Results of a Case-Control Study," Blood, Vol. 96, No. 7, 2000, pp. 2364-2368.

[13] D. C. Valla and B. Condat, "Portal Vein Thrombosis in Adults: Pathophysiology, Pathogenesis and Management," Journal of Hepatology, Vol. 32, No. 5, 2000, pp. 865-871. doi:10.1016/S0168-8278(00)80259-7

[14] M. den Heijer, T. Koster, H. J. Blom, et al., "Hyperhomocysteinemia as a Risk Factor for Deep-Vein Thrombosis," New England Journal of Medicine, Vol. 334, No. 21, 1996, pp. 759-762. doi:10.1056/NEJM199603213341203

[15] M. A. Avila, C. Berasain, L. Torres, et al., "Reduced mRNA Abundance of the Main Enzymes Involved in Methionine Metabolism in Human Liver Cirrhosis and Hepatocellular Carcinoma," Journal of Hepatology, Vol. 33, No. 6, 2000, pp. 907-914. doi:10.1016/S0168-8278(00)80122-1

[16] M. R. Gomez, E. S. Garcia, D. L. Lacomba, I. Marchante, L. Grande, M. C. Fernandez, "Anti-phospholipid Anti- 
bodies are Related to Portal Vein Thrombosis in Patients with Liver Cirrhosis," Journal of Clinical Gastroenterology, Vol. 31, No. 3, 2000, pp. 237-240. doi:10.1097/00004836-200010000-00011

[17] F. Dentali, M. Galli, M. Gianni and W. Ageno, "Inherited Thrombophilic Abnormalities and Risk of Portal Vein Thrombosis: A Meta-analysis," Thromb Haemost, Vol. 99, No. 4, 2008, pp. 675-682.

[18] G. J. Webster, A. K. Burroughs, S. M. Riordan, "Review Article: Portal Vein Thrombosis - New Insights into Aetiology and Management," Alimentery Pharmacology and Therapeutics, Vol. 21, No. 1, 2005, pp. 1-9. doi:10.1111/j.1365-2036.2004.02301.x

[19] J. P. Duffy, J. C. Hong, D. G. Farmer, et al., "Vascular Complications of Orthotopic Liver Transplantation: Experience in More than 42,000 Patients," Journal of the American College of Surgeons, Vol. 208, No. 5, 2009, pp. 896-903.

[20] E. P. Molmenti, et al., "Thrombendvenectomy for Organized Portal Vein Thrombosis at the Time of Liver Transplantation," Annals of Surgery, Vol. 235, 2002, pp. 292-296. doi:10.1097/00000658-200202000-00019

[21] J. Dumortier, et al., "Eversion Thrombectomy for Portal Vein Thrombosis during Liver Transplantation," American Journal of Transplantation, Vol. 2, No. 10, 2002, pp. 934-938. doi:10.1097/00000658-200202000-00019

\section{Abbreviations}

ESLD: end stage liver disease; HCC: hepatocellular carcinoma; HCV: hepatitis C; MELD: model for end stage
[22] J. Neugarten and S. R. Silbiger, "The Impact of Gender on Renal Transplantation," Transplantation, Vol. 15, 1994, pp. 1145-1152. doi:10.1097/00007890-199412150-00001

[23] J. Neugarten, T. Srinivas, V. Tellis, S. R. Silbiger and S. Greenstein, "The Effect of Donor Gender on Renal Allograft Survival," Journal of the American Society of Nephrology, Vol. 7, No. 2, 1996, pp. 318-324.

[24] P. Vereerstraeten, M. Wissing, L. DePauww and D. K. Abramowicz, "Male Recipients of Kidneys from Female Donors are at Increased Risk of Graft Loss from Both Rejection and Technical Failure," Clinical Transplantation, Vol. 13, 1999, pp. 181-186. doi:10.1034/j.1399-0012.1999.130205.x

[25] M. Zeier, B. Dohler, G. Opelz and E. Ritz, "The Effect of Donor Gender on Graft Survival," Journal of the American Society of Nephology, Vol. 13, No. 10, 2002, pp. 2570-2576. doi:10.1097/01.ASN.0000030078.74889.69

[26] M. Hertl, J. F. Markmann, P. S. Russell and H. Yeh, "Liver Transplantation," Merck Manual, Sept 2008.

[27] D. Kahn, J. S. Gavaler, L. Makowka, D. H. van Thiel, "Gender of Donor Influences Outcome after Orthotopic Liver Transplant in Adults," Digestive Diseases and Sciences, Vol. 38, No. 8, 1993, pp. 1485-1488. doi:10.1007/BF01308608

liver disease; NASH: non-alcoholic steatohepatitis; OLT: orthotopic liver transplant; PSC: primary sclerosing cholangitis; PVT: portal vein thrombosis. 